九州大学学術情報リポジトリ

Kyushu University Institutional Repository

\title{
Impacts of Amplitude Modulation of RF Discharge Voltage on the Growth of Nanoparticles in Reactive Plasmas
}

Kamataki, Kunihiro

Center for Research and Advancement in Higher Education, Kyushu University

Miyata, Hiroshi

Department of Electric and Electronics, Graduate School of Information Science and Electrical Engineering, Kyushu University

Koga, Kazunor i

Department of Electric and Electronics, Graduate School of Information Science and Electrical Engineering, Kyushu University

Uchida, Gi ichiro

Department of Electric and Electronics, Graduate School of Information Science and Electrical Engineering, Kyushu University

他

ht tp://hdl. hand le. net/2324/26600

出版情報: Applied Physics Express. 4 (10)，pp.105001(1)-105001(3)，2011-10. 応用物理学会 バージョン：

権利関係: (C) 2011 The Japan Society of Applied Physics 


\section{Impacts of Amplitude Modulation of rf Discharge Voltage on the Growth of Nanoparticles in Reactive Plasmas}

$\underline{\text { Kunihiro Kamataki, Hiroshi Miyata }}{ }^{1}$, Kazunori Koga ${ }^{1}$, Giichiro Uchida ${ }^{1}$, Naho Itagaki ${ }^{1}$, and Masaharu Shiratani ${ }^{1}$

Center for Research and Advancement in Higher Education, Kyushu University,744 Motooka, Fukuoka, Japan

${ }^{1}$ Department of Electric and Electronics Graduate School of Information Science and Electrical Engineering, Kyushu University, 744 Motooka, Fukuoka, Japan

*kamataki@rche.kyushu-u.ac.jp

We have investigated the effects of plasma fluctuation on the growth of nanoparticles in capacitively-coupled rf discharges with amplitude modulation (AM). Nanoparticles grow more slowly for higher AM levels, which causes the density of nanoparticles to increase by $100 \%$ and their size to decrease by $23 \%$. The increase in the number of radicals for nucleation and nanoparticles by AM is thought to cause a decrease in the radical flux for a nanoparticle because the rate of increase in the number of radicals is smaller than that of nanoparticles. Therefore, this causes the generation of a large amount of nanoparticles with small sizes. 
Studies focused on reducing the dimensions of semiconductor structures ${ }^{1)}$ to the nanometer regime have led to a fascinating class of novel materials that exhibit quantum confinement characteristics. These materials are referred to as nanoparticles or quantum dots. Although such nano-composite materials with quantum dots allows us to fabricate high-efficiency optoelectronic devices, ${ }^{2-4)}$ efficient methods of synthesizing large numbers of small nonagglomerated nanoparticles with a narrow size distribution are required. Plasma processing can be a very useful tool for synthesizing such nanoparticles in the gas phase of reactive plasmas. ${ }^{5-11)}$ For faster development of fabrication methods for nano-materials, the effects of plasma fluctuations on the growth of nanoparticles in reactive plasmas must be taken into account because plasma fluctuations affect nanoparticles in dust plasmas. ${ }^{12-16)}$ Investigation of the effects leads to the control of the growth of nanoparticles and to the improvement of the fabrication technology of nano-materials. Matsuoka et al. found the suppression of particle growth by controlling the gas flow in silane RF discharges. ${ }^{17)}$ Koga et al. succeed in the fast transport of nano-particles using AM method which was applied as the square wave modulation only at the end of the discharge time. ${ }^{18,19)}$ Kashihara et al. reported the suppression of particle generation using a sine-wave modulated rf plasma, which method switched rf power on-off by sine wave instead of pulse-wave modulation for more stable operation. ${ }^{20)}$ However, the mechanisms of effects of plasma fluctuations on the growth of nanoparticles in reactive plasmas have not yet been reported. We have already confirmed that new AM method, which was applied as sine-wave modulation at the all discharge time (refer to Fig.2(d)) for the investigation above-mentioned effects, can control the plasma fluctuation level in reactive plasma. ${ }^{21)}$

In the present paper, we report the effects of plasma fluctuations on the growth of nanoparticles in reactive plasmas using an in-situ laser-light scattering (LLS) method. Moreover, we clarified the mechanism of the effects and succeed in the production of a higher density of smaller nanoparticles than in the case of using the conventional technique ${ }^{9)}$.

The present study is limited to the investigation of time-averaged effects. The dynamics of the interaction between plasma fluctuations and growth will be reported in a forthcoming article.

Experiments were performed using a capacitively coupled rf discharge reactor with an LLS system, as shown in Fig. 1. ${ }^{21)}$ The size of the reactor and electrodes are same as that in Ref. 21. The reactor was evacuated to a base pressure of less than $10^{-3} \mathrm{~Pa}$ with a rotary pump and a diffusion pump. The flow rates of $\mathrm{Si}\left(\mathrm{CH}_{3}\right)_{2}\left(\mathrm{OCH}_{3}\right)_{2}$ and $\mathrm{Ar}$ were 0.2 and $40 \mathrm{sccm}$, respectively. The total gas pressure in the reactor was 133 $\mathrm{Pa}$. The temperature of the reactor wall was maintained at $373 \mathrm{~K}$ in order to avoid the liquefaction of $\mathrm{Si}\left(\mathrm{CH}_{3}\right)_{2}\left(\mathrm{OCH}_{3}\right)_{2}$. In order to generate nanoparticles, we sustained a discharge by applying 150 peak-to-peak voltage $V_{\mathrm{pp}}$ of $60 \mathrm{MHz}$ to the powered electrode for a discharge period of $T=8 \mathrm{~s}$. The corresponding discharge power was $30 \mathrm{~W}$. The self-bias voltage $V_{\mathrm{dc}}$ was $0.4 \mathrm{~V}$. The plasma parameters of electron density $n_{\mathrm{e}}$ and floating potential $V_{\mathrm{f}}$ were measured using an rf-compensated Langmuir probe. The typical electron density $n_{\mathrm{e}}$ was approximately $10^{10}-10^{11} \mathrm{~cm}^{-3}$. 
The growth of nanoparticles was monitored using the in-situ LLS method. The size and density of nanoparticles were deduced from their coagulation process, which took place after turning off the discharge using the procedure described in ref. 22. The spatio-temporal evolution of the LLS intensity between the upper grounded electrode and the powered rf electrode was obtained using an ICCD camera (Hamamatsu Photonics) with a frame rate of $30 \mathrm{~s}^{-1}$. The observed region was an area of $\mathrm{r}=0-32 \mathrm{~mm}$ and $\mathrm{z}=0-20 \mathrm{~mm}$, where $(r, z)=(0 \mathrm{~mm}, 0 \mathrm{~mm})$ was the center of the upper surface of the powered electrode.

For AM discharges, the discharge voltage was modulated as shown in Figs. 2(a) and 2(b) with a sine waveform having a modulation frequency $f_{\mathrm{AM}}$ of $10 \mathrm{kHz}$. Figures 2(c) and 2(d) show the typical time evolution of the floating potential without and with AM, respectively. Thus, it is found that the floating potential can be controlled by AM of the rf discharge voltage.

Figure 3 shows the time evolution of the LLS intensity with and without AM at the plasma/sheath boundary region near the upper grounded electrode for three modulation levels of 10, 20, and 30\%. It can be seen that although the LLS intensities under each condition increase with time, they are suppressed by the presence of AM. At $t=8 \mathrm{~s}$, the LLS intensity for the $30 \%$ AM case is 0.4 times that without AM. These results show that AM of the rf discharge voltage suppresses the growth of nanoparticles.

In order to clarify the growth suppression mechanism, the size and density of nanoparticles were determined based on the LLS intensities. Figure 4 shows the dependence of the size and density of nanoparticles on the AM level, at $(r, z)=(0.0 \mathrm{~mm}, 17.5 \mathrm{~mm})$ at the plasma/sheath boundary region near the upper grounded electrode. The nanoparticle density increases by $100 \%$ from $3 \times 10^{9} \mathrm{~cm}^{-3}$ for the $0 \%$ AM level to $6 \times 10^{9} \mathrm{~cm}^{-3}$ for the $30 \%$ AM level. Moreover, the nanoparticle size decreases by $23 \%$ from $12.5 \mathrm{~nm}$ for the $0 \%$ AM level to $9.7 \mathrm{~nm}$ for the $30 \%$ AM level. In ref. 9, Watanabe controlled the size and density of nanoparticles by varying the rf discharge power and/or discharge duration. Here, we show that AM of the rf discharge voltage is another method of tuning the nanoparticle size and density.

Figure 5 shows the dependence of the spatially integrated density and the volume fraction of nanoparticles on the AM level. Here, the integration is performed over the range $r=0-32 \mathrm{~mm}$, and the volume fraction is a volume occupied by nano-particles per unit volume, which is defined as $\pi n_{\mathrm{p}}\left(d_{\mathrm{p}}\right)^{3} / 6$. The spatial integrated volume fraction is related to the number of radicals for the nucleation of nanoparticles. This result shows that both the number of nanoparticles and radicals for nucleation increase with the AM level.

Figure 6 shows the AM level dependence of the ratio of the number of radicals to the number of nanoparticles. The number of radicals was determined based on the abovementioned integrated volume fraction of nanoparticles under the assumption that all the radicals that contribute to nanoparticle growth are $\mathrm{SiH} x(x=0-3)$ and that the nanoparticles are predominantly composed of $\mathrm{Si}^{22}{ }^{22} \mathrm{We}$ focus on these qualitative trends of the relationship between the AM level and the ratio of the number of radicals to the number of nanoparticles. This result shows that the ratio decreases as the AM level increases. The 
mechanism by which AM of the rf discharge in a reactive plasma affects nanoparticle nucleation and growth is thought to be as follows. The number of radicals for nucleation of nanoparticles increases with AM level, which decreases the radical flux to nanoparticles because the rate of increase of the number of nanoparticles is higher than that of radicals with increasing AM level. This leads to the suppression of nanoparticle growth and the generation of a high density of small nanoparticles.

We now introduce a nanoparticle growth model in order to investigate the dependence of the growth rate on the density of nanoparticles under the experimental conditions of the present study. The growth rate is proportional to the radical density $n_{\mathrm{r}}$. In this analysis, a simple growth model is evaluated. During discharge with AM, radicals are produced at a constant rate and are eliminated when nanoparticles collide with the reactor wall. The emission intensity of $\operatorname{ArI}(\lambda 1=750.4 \mathrm{~nm})$ obtained by OES measurement is approximately the same as that when the AM level increased from 10 to $30 \%$ (not shown). In the proposed model, the growth rate is expressed as $\mathrm{d} n_{\mathrm{r}} / \mathrm{dt}=$ [radical production rate]-[radical deletion rate] (1), where [radical deletion rate] is proportional to $n_{\mathrm{r}}\left(A_{\mathrm{w}} / \tau_{\mathrm{w}}+A_{\mathrm{p}} / \tau_{\mathrm{p}}\right)$, which is time-independent. Here, the total surface area of the characteristic plasma column volume, $A_{\mathrm{w}}$, is $6 \mathrm{Lw}_{\mathrm{w}}^{2}$, where $L_{\mathrm{w}}$ is the characteristic plasma column length, and the total surface area of all the nanoparticles in the abovementioned volume, $A_{\mathrm{p}}$, is $4 \pi\left(d_{\mathrm{p}} / 2\right)^{2} n_{\mathrm{p}} L_{\mathrm{w}}{ }^{3}$. The characteristic times for radical diffusion to the reactor wall and to nanoparticles are defined as $\tau_{\mathrm{w}}=L_{\mathrm{w}} / \mathrm{D}$ and $\tau_{\mathrm{p}}=L_{\mathrm{p}} / \mathrm{D}$, respectively, where $D$ is the diffusion coefficient of radicals and the distance between nanoparticles is $L_{\mathrm{p}}=n_{\mathrm{p}}{ }^{-1 / 3}$. In the steady state, with $\mathrm{d} / \mathrm{dt}=0$, the solution of eq. (1) is obtained as $0 \sim n_{\mathrm{r}}\left(A_{\mathrm{w}} / \tau_{\mathrm{w}}+A_{\mathrm{p}} / \tau_{\mathrm{p}}\right)$. Then, $n_{\mathrm{r}} \propto 1 /\left(A_{\mathrm{w}} / \tau_{\mathrm{w}}+A_{\mathrm{p}} / \tau_{\mathrm{p}}\right)$. Figure 7 shows the growth rate of nanoparticles as a function of the density of nanoparticles for different values of $L_{\mathrm{w}}\left(d_{\mathrm{p}}=10 \mathrm{~nm}\right)$, together with the experimental results for $L_{\mathrm{w}}=0.1 \mathrm{~m}$. There are two growth rate regions. In the first region, the growth rate of nanoparticles does not depend on the nanoparticle density because the loss of radicals to the reactor wall is dominant. In the second region, the growth rate decreases with increasing nanoparticle density because the loss of radicals to nanoparticles surpasses that to the reactor wall. The experimental results obtained for the second region agree well with the theoretical results. For nanoparticle production in the absence of a plasma, a higher density of nanoparticles leads to faster growth due to the higher agglomeration rate and larger variance in the nanoparticle size distribution. On the other hand, in the production of nanoparticles with plasmas, most (or some) nanoparticles are negatively charged, and their agglomeration is suppressed due to electrostatic repulsion. Therefore, in the second nanoparticle production region in reactive plasmas, a negative feedback regarding the growth of nanoparticles occurs. As a result, reactive plasmas are promising as methods of producing a large quantity of small nanoparticles with a narrow size dispersion.

In conclusion, we herein investigated the effects of AM on the growth of nanoparticles in reactive CVD plasmas. The growth of nanoparticles during rf discharges was decelerated as the induced plasma fluctuation level increased. The density of nanoparticles increased by $100 \%$ and the nanoparticle size 
decreased by $23 \%$ when the AM level increased from 0 to $30 \%$. The mechanism behind these results is thought to be as follows. 1) The number of radicals for the nucleation of nanoparticles increases with AM level. 2) This leads to a decrease in the radical flux to nanoparticles because the loss of radicals to nanoparticles surpasses that to the reactor wall. 3) This suppresses the growth of nanoparticles and eventually leads to the production of a high density of small nanoparticles. The proposed AM method was used to control the fluctuation level of the plasma parameters and allowed a large amount of small nanoparticles to be generated under constant input power.

\section{Acknowledgements}

The authors would like to thank Dr. S. Iwashita for helpful discussions. The present study was supported in part by a Grant-in-Aid for Scientific Research on Innovative Areas (No. 2109) from the Ministry of Education, Culture, Sports, Science, and Technology, Japan. 
1) ITRS Teams 2010 International Technology Roadmap for Semiconductors http://www.itrs.net/home.html.

2) A. J. Nozik: Physica E 14 (2002) 115.

3) J. M. Elzerman, R. Hanson, J. S. Greidanus, L. H. Willems van Beveren, S. De. Franceschi: L. M. K. Vandersypen, S. Tarucha, and L. P. Kouwenhoven, Phys. Rev. B 67 (2003) 161308(R).

4) V. I. Klimov, A. A. Milhailovsky, S. Xu, A. Malko, J. A. Hollingsworth, C. A. Leatherdale, H. J. Eisler, and M. G. Bawendi: Science, 290 (2000) 314.

5) Y. Watanabe, M. Shiratani, Y. Kubo, I. Ogawa, and S. Ogi: Appl. Phys. Lett. 53 (1988) 1263.

${ }^{6)}$ M. Shiratani, T. Fukuzawa, and Y. Watanabe: Jpn. J. Appl. Phys. 38 (1999) 4542.

${ }^{7)}$ S. Nunomura, M. Shiratani, K. Koga, M. Kondo, and Y. Watanabe: Phys. Plasmas, 15 (2008) 080703.

${ }^{8)}$ S. Nunomura, K. Koga, M. Shiratani, Y. Watanabe, Y. Morisada, N. Matsuki, and S. Ikeda: Jpn. J. Appl. Phys., 44 ( 2005) L1509.

9) Y. Watanabe: J. Phys. D 39 (2006) R329.

${ }^{10)}$ S. Nunomura, M. Shiratani, K. Koga, M. Kondo, and Y. Watanabe: Phys. Plasmas, 15 (2008) 080703.

11) F. X. Bronold, H. Fehske, H. Kersten, and H. Deutsch: Phys. Rev. Lett. 101 (2008) 175002.

12) G. Praburam and J. Goree: Phys. Plasmas, 3 (1996) 1212.

${ }^{13)}$ D. Samsonv and J. Goree: Phys. Rev. E, 59 (1999) 1047.

14) V. E. Fortov, A. V. Ivlev, S. A. Khrapak, A. G. Khrapak, and G. E. Morfill: Phys. Rep. 421 (2005) 1.

15) V. Nosenko and J. Goree: Phys. Rev. Lett. 93 (2004) 155004.

16) S. Ratynskaia, M. De Angeli, U. de Angelis, C. Marmolino, G. Capobianco, M. Lontano, E. Lazzaro, G.

E. Morfill, and G. Gervsini: Phys. Rev. Lett. 99 (2007) 075002.

17) Y. Matsuoka, M. Shiratani, T. Fukuzawa, Y. Watanabe, and K. -S. Kim: Jpn. J. Appl. Phys. 38 (1999) 4556.

${ }^{18)}$ K. Koga, S. Iwashita, and M. Shiratani: J. Phys. D: Appl. Phys. 40 (2007) 2267.

19) M. Shiratani, K. Koga, S. Iwashita, and S. Nunomura: Faraday Discuss. 137 (2008) 127.

${ }^{20)}$ N. Kashihara, H. Setyawan, M. Shimada, Y. Hayashi, C. S. Kim, K. Okuyama and S. Winnardi: J. Nano Res. 8 (2006) 395-403.

${ }^{21)}$ K. Kamataki, H. Miyata, K. Koga, G. Uchida, N. Itagaki, D. Yamashita, H. Matsuzaki, and M. Shiratani: Proc. IEEE Region 10 Int. Conf. T2-19 (2010) 1943.

${ }^{22)}$ S. Nunomura, M. Kita, K. Koga, M. Shiratani, and Y. Watanabe: J. Appl. Phys. 99 (2006) 083302. 
Figure Captions

Fig.1. Experimental setup. In order to monitor the growth of nanoparticles, the nanoparticles are illuminated by SHG of CW YAG laser light $(\lambda=532 \mathrm{~nm}, 2.0 \mathrm{~W})$, and the scattering light is detected using an ICCD camera.

Fig.2. Time evolution of $V_{\mathrm{pp}}$ and $V_{\mathrm{f}}$ of pure Ar discharges without [(a), (c)] and with [(b), (d)] AM. The modulation level is $40 \%$, and $f_{\mathrm{AM}}$ is $10 \mathrm{kHz}$.

Fig.3. Time evolution of the LLS intensity with and without AM at the plasma/sheath region near the upper grounded electrode for AM levels of 10,20 , and $30 \%$ and $f_{\mathrm{AM}}=10 \mathrm{kHz}$.

Fig.4. AM level dependence of the density and size of nanoparticles at $(\mathrm{r}, \mathrm{z})=(0.0 \mathrm{~mm}, 17.5 \mathrm{~mm})$.

Fig.5. AM level dependence of spatial integrated density and volume fraction of nanoparticles.

Fig.6. AM level dependence of the ratio of the number of radicals to the number of nanoparticles.

Fig.7. Dependence of the growth ratio on the density of nanoparticles from the nanoparticle growth model. The circles indicate experimental results $\left(L_{\mathrm{w}}=0.1 \mathrm{~m}\right)$. 


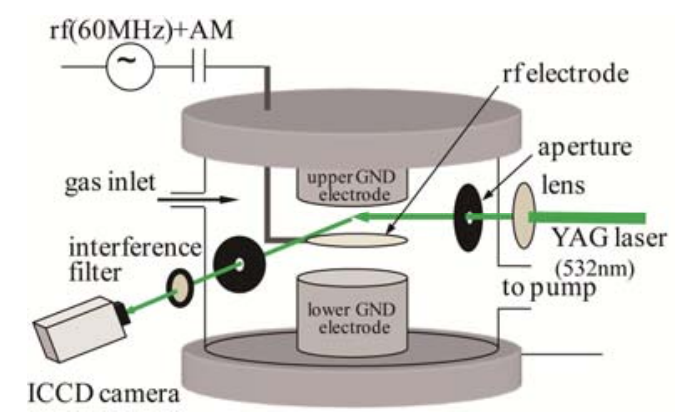

Fig.1. K. Kamataki et al. 


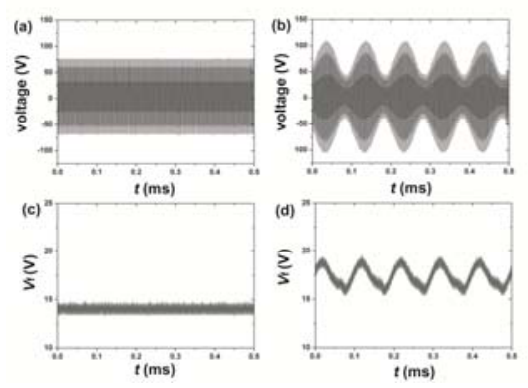

Fig. 2. K.Kamataki et al. 


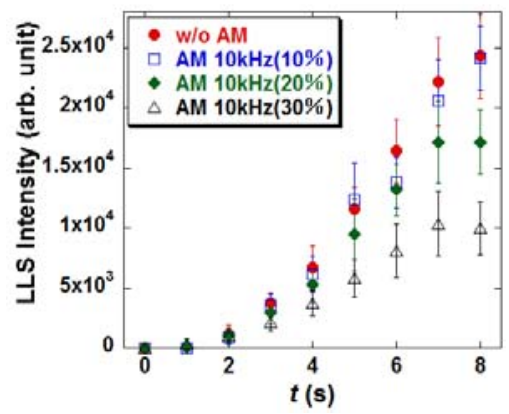

Fig. 3. K.Kamataki et al. 


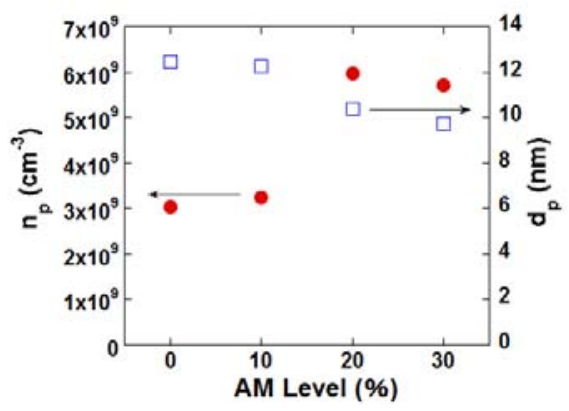

Fig. 4. K.Kamataki et al. 


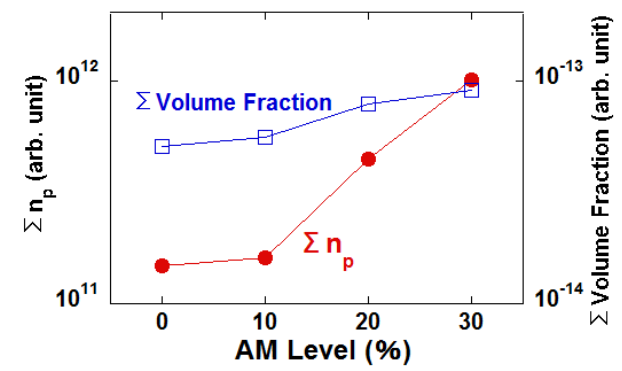

Fig. 5. K.Kamataki et al. 


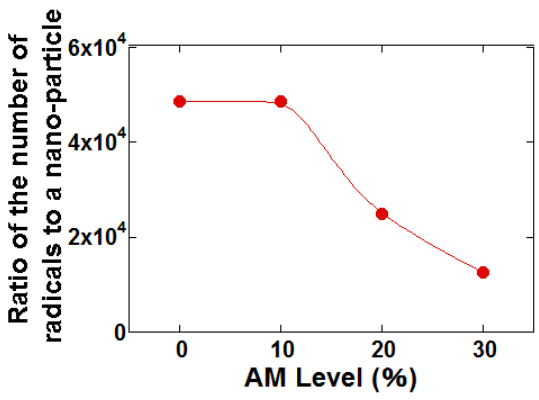

Fig. 6. K.Kamataki et al. 


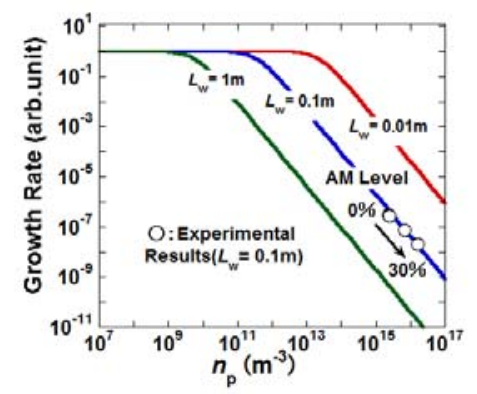

Fig.7. K.Kamataki et al. 